\title{
Protée
}

\section{N'y a-t-il point quelque danger à contrefaire Molière?}

\section{Dominique Lafon}

Volume 27, numéro 1, 1999

La Mort de Molière et des autres

URI : https://id.erudit.org/iderudit/030535ar

DOI : https://doi.org/10.7202/030535ar

Aller au sommaire du numéro

Éditeur(s)

Département des arts et lettres - Université du Québec à Chicoutimi

ISSN

0300-3523 (imprimé)

1708-2307 (numérique)

Découvrir la revue

Citer cet article

Lafon, D. (1999). N'y a-t-il point quelque danger à contrefaire Molière? Protée, 27(1), 21-28. https://doi.org/10.7202/030535ar

\section{Résumé de l'article}

Comment rendre compte du fétichisme d'un mythe, nourri de plus de légendes que de faits avérés, quand il prend pour forme une iconographie qui dissimule ses sources dans l'éclatement de séquences volontairement illisibles? En dessinant la mosaïque des références, sans doute, mais aussi en confrontant le discours postmoderne aux épitaphes du XVII ${ }^{\mathrm{e}}$ siècle, qui ne célèbrent rien d'autre que le vertige du comédien pris au piège de son interprétation. C'est dans cette confrontation que se révèle le véritable enjeu de La Mort de Molière, non point seulement filmée, mais rejouée par Robert Wilson qui exorcise la mort de l'acteur, victime impénitente du même exercice, dresse la stèle d'une agonie exemplaire, pour ériger, avant l'heure, le tombeau de l'artiste contemporain. 


\title{
N'Y A-T-IL POINT QUELQUE DANGER À CONTREFAIRE MOLIÈRE?
}

DOMINIQUE LAFON

\author{
Dies ist kein Gedicht über Molière. Über Molière kann man kein \\ Gedicht schreiben. Molière ist kein poetischer Gegenstand. Molière ist \\ ein Objekt der Medizin. (H. Müller, texte de présentation)
}

On pardonnera à la germaniste que je fus jadis d'avoir renoué avec quelques souffrances estudiantines en lisant la version allemande des premières phrases du texte composé par Müller sur le «sujet» qu'est la mort de Molière. En vérité, ce texte liminaire fut écrit durant l'automne 1993 non seulement sur Molière, mais aussi sur le film tourné par Wilson, quelques mois plus tôt, le texte des séquences ayant été établi une année plus tard.

Si Molière n'est pas un sujet de poésie, sa mort le fut pour son époque qui lui érigea de nombreux tombeaux poétiques. Le terme de mausolée rendrait mieux compte, sans doute, d'un phénomène que Roger Duchêne décrit comme suit:

Nulle mort d'écrivain du XVIIe siècle n'a été l'occasion d'un tel déferlement d'épitaphes et de poèmes en tous genres: une centaine dont plusieurs en latin. Sa fin soudaine, presque sur scène, a fait d'un comédien célèbre et d'un auteur controversé un mythe vivant autour duquel se sont affrontés les défenseurs et les ennemis du théâtre, les dévots et les libertins, les tenants de la grande comédie et les amateurs de farce. (1998: 670)

Invitée, comme moliériste, à écrire sur un tombeau contemporain, modeste dalle funéraire de quarante-cinq minutes, on comprendra que j'aie d'abord interprété le paradigme des négations du poème d'ouverture, du poème générique dans tous les sens du terme, début d'un film mais aussi mise en question du genre de l'épitaphe, comme une dénégation, voire une méconnaissance des origines de la constitution d'un mythe. La mise en garde de l'exergue devenait paradoxalement une invite à convoquer les épitaphes, puis les pièces de théâtre, qui voulurent tirer la leçon, fixer l'image des circonstances de la mort de Molière; florilège contrapuntique inattendu pour servir à l'analyse d'une œuvre résolument inscrite dans la modernité. Le collage de textes divers, et à première vue immotivés, sur lequel la vidéo repose en grande part, autorisait sa confrontation avec des textes contemporains à l'événement éponyme. 
La Mort de Molière de Robert Wilson n'est pas, dirat-on, une œuvre de circonstance. Pourtant sa gestation, son élaboration furent tributaires de bien des circonstances extérieures. Un changement de ministre de la culture, la réunion des deux Allemagnes et, plus significativement, un hasard, celui dont les étoiles ne se soucient pas.

Hasard, l'accrochage d'une toile de style pompier dans le bureau de l'Administrateur de la ComédieFrançaise, qui représente Molière assis dans un grand fauteuil, la tête rejetée en arrière. Symétriquement agenouillées de part et d'autre des jambes du personnage central, deux religieuses. Il s'agit d'un tableau de Wafflard, peint en 1810 (Lorcey, 1980: 223), qui met en scène l'Histoire, la présence, dans la maison de Molière, au jour de sa mort, de deux religieuses qui quêtaient pour le Carême et qu'on avait hébergées, suivant les us de l'époque. Les deux bustes qu'on devine à la gauche du tableau sont certainement ceux de Plaute et Térence, dramaturges auxquels les panégyristes des épitaphes associent ordinairement Molière. À commencer par le plus célèbre d'entre eux, La Fontaine:

Sous ce tombeau gisent Plaute et Térence,
Et cependant le seul Molière y gît.
Leurs trois talents ne formaient qu'un esprit
Dont le bel art réjouissait la France.
Ils sont partis, et j'ai peu d'espérance
De les revoir, malgré tous nos efforts.
Pour un long temps, selon toute apparence,
Térence, et Plaute, et Molière sont morts.

(Donneau de Visé, 1879: 49)

$\mathrm{Si}$, dans la vidéo, les religieuses du tableau de Wafflard apparaissent dès les premières images, la référence humaniste n'y tient pas une grande place. Comme le tableau, cependant, elle donnera à voir les manuscrits de l'auteur, objets mythiques dont la disparition demeure la pierre d'achoppement de la légende moliéresque.

Ce tableau, qui fut à l'origine du projet wilsonien, s'inspire, quant à lui, d'une des branches tardives de la biographie moliéresque, puisque la présence des deux religieuses intervient dans les récits de la mort de Molière pour la première fois en 1705 , chez Grimarest, qui fut un des auteurs de référence pour le recherchiste de Wilson, Jan Linders. Le récit des tout derniers instants de Molière, qui n'est pas cité dans la vidéo où il eût été redondant, est très révélateur de la fonction de ces deux personnages étrangers à l'univers domestique de l'acteur:

Il resta assisté de deux soeurs religieuses [Armande et Baron sont partis.] Elles lui donnèrent à ce dernier moment de sa vie tout le secours édifiant que l'on pouvait attendre de leur charité, et il leur fit paraitre tous les sentiments d'un bon chrétien et toute la résignation qu'il devait à la volonté du Seigneur. Enfin il rendit l'esprit entre les bras de ces deux bonnes soeurs; le sang, qui sortait par la bouche en abondance, l'étouffa. Ainsi quand sa femme et Baron remontèrent, ils le trowvèrent mort.

(Grimarest, La Vie de Monsieur de Molière [1705], cité dans Duchêne, 1998: 662-663)

Ce récit, que Grimarest prétend tenir de Baron luimême, peut se lire comme une enluminure si on le compare au texte de la requête d'Armande Béjart à l'Achevêque de Paris, pour demander la permission de donner une sépulture ecclésiastique à Molière:

Ce considéré, Monseigneur, et attendu [...] que ledict deffunct a demandé auparavant que de mourir un prestre pour estre confessé, qu'il est mort dans le sentiment d'un bon chrestien, ainsi qu'il a témoigné en presence de deux dames religieuses demeurant en la mesme maison, d'un gentilhomme nommé Couton, entre les bras de qui il est mort et de plusieurs autres personnes. (Jürgens et Maxfield-Miller, 1963:551)

La valeur compensatoire de la présence des deux sœurs clarisses d'Annecy est manifeste; ce sont des figures tutélaires qui recueillent le rachat du pécheur. Le tableau du XIX entérine le chromo, l'exacerbe même, en agenouillant les deux religieuses aux pieds d'un Molière qui semble les bénir.

Les auteurs des épitaphes rédigées dans les jours et les mois qui suivirent la mort de Molière ne pouvaient avoir recours à ces deux figures, commodes truchements dont ils ignoraient l'existence. Mais la violence des accusations d'impiété dont ils se font 
l'écho explique pourquoi Grimarest leur attribue un rôle décisif.

Que disent ces épitaphes?

$\mathrm{Ci}$ git qui toujours se moquoit,

Qui pour plaire aux grands, se piquoit

D'une impiété sans seconde;

Nul ne fut épargné de lui;

Le bouffon sçait bien aujourd'hui

Si l'on se raille en l'autre monde.

(Monval, 1969: 33)

Molière est mort subitement;

Le diable l'a pris brusquement

Et le tient enfermé dans sa grotte profonde;

Son sort était égal pendant qu'il a vécu;

Car, étant jaloux et cocu

Il était damné dans ce monde. (Loc. cit.)

Molière repose en ce lieu,

Qui par des ouvres exécrables

Se mit si mal auprès de Dieu

Qu'il est très bien auprès des diables. (Ibid., p. 34)

J'attaque impunément le plus haut caractère

Des Roys et des dévôts, des marquis, du vulgaire;

J'ay de tous les états découvert le mystère:

Joüant le medecin, je me suis échoüé.

Je meurs sans medecin, sans prêtre, sans notaire:

J'ay joüé la Mort même et la Mort m'a joüé. (Ibid., p. 16)

Cy gît le comique Moliere

Sans sonner, sans chanter, sans lumière,

Enterré clandestinement

Dedans le coin d'un cimetiere. (Ibid., p. 51)

Et Wilson? Wilson consacre un long plan de la dixième séquence, séquence de la mort, à un cercle de huit religieuses qui jouent, pour reprendre les termes de Jan Linders, «a strange ritual»; rituel gestuel où l'on peut parfois identifier le geste de «battre sa coulpe». Mais le plan suivant qui les montre s'allongeant face contre terre, comme lors de la prise de voile, les sacrifie totalement au mythe auquel, symboliquement, elles s'unissent. La problématique religieuse s'abîme dans la prosternation, et avec elle les périls que fait encourir la dénonciation de la tartufferie. L'acteur est un pauvre homme, pas un moraliste.

La présence de Galilée au chevet de Molière pourrait donner à voir le pauvre homme en penseur. Elle est peut-être le fruit d'un autre hasard, plus secret il est vrai, mais qui éclaire encore l'image par le biais d'une autre circonstance: la présence dans le secrétariat général de la Comédie-Française d'un portrait de Galilée, œuvre d'un inconnu, signalée dans le catalogue du Musée de la Comédie-Française, édité par René Delorme en 1878.

Ceci n'est pas un tableau, même s'il en prend le titre. Le résultat, ici La Mort de Molière, dépend d'autres circonstances que le cours des étoiles politiques et l'observation des toiles accrochées au mur...

Ceci n'est pas une pipe. Ceci est un poème sur Molière. Das ist ein Gedicht über Molière. Le poème n'est pas un film. Le poème comme le film observent un mourant, un acteur, "bei der Arbeit», au travail.

Ceci n'est pas une communication savante. L'universitaire observe le travail d'un vidéaste qui recompose un catalogue de Musée, fasciné par le cercueil de verre qui protège le fauteuil de Molière et les images qui l'entourent. Dans ce catalogue pourrait figurer un tableau ou un document relatif à Racine, dont on peut supposer, même si cela reste à vérifier, qu'il représente Racine lisant Plutarque au Roi sur le champ de bataille.

Ceci n'est pas la mort de Molière, mais l'image au travail ou le travail de l'image d'un acteur qui joue la mort d'un homme qui s'appelait Molière.

Robert Wilson n'est pas Molière. Molière n'était pas un médecin.

Molière était un acteur qui mourut d'avoir, bei der Arbeit, contrefait le mort et le médecin dans une pièce intitulée Le Malade imaginaire. Les épitaphes nous le font bien connaître, qui déclinent les dangers de la contrefaçon:

Ce n'était qu'une comédie

Où Molière faisoit le malade et le mort. 
La mort, en étant avertie,

Voulut joüer son rôle et faire sa partie.

Par forme d'épisode elle parut d'abord;

Mais devenant jalouse enfin de sa copie,

Fit que l'acte dernier décida de son sort,

Et changea le comique en une tragédie.

Molière, appellois-tu Malade imaginaire

Celuy qui meurt réellement?

Peut-être qu'à ton jugement

La mort n'était qu'une chimère,

Malgré le commun sentiment

Qui nous enseigne le contraire. (Monval, 1969: 25)

Cy gît un illustre bouffon

Qui n'a pû si bien contrefaire

Le Malade imaginaire

Qu'il a fait le mort pour de bon. (Ibid., p. 15)

Cy gist qui dans la comédie

Pour dernier acte de sa vie,

Voulut représenter le mort:

Pour mieux jouer ce personnage,

D'un vray mort prenant le visage,

D'un sommeil éternel à la fin il s'endort. (Ibid., p. 27)

Robert Wilson n'est pas Molière. Robert Wilson est un metteur en scène original qui joue le rôle de Molière mourant sur un collage de textes conçu par un dramaturge allemand, dont la veillée mortuaire s'acheva par la projection d'une vidéo intitulé La Mort de Molière. Ceci n'est pas la mort de Müller.

Ceci n'est pas la mort de Molière.

Ceci n'est pas un documentaire. On n'apprend rien du visionnement de la vidéo de la biographie de Molière. Seul le générique ou la lecture du synopsis, présenté par Jan Linders dans Performance Research, permet d'identifier la présence de membres de la troupe, tel La Grange et La Thorillière, ou celle encore plus improbable de Chapelle, qui fut peut-être le condisciple de Molière. Il y a aussi quelque incongruité à donner à la fille de Molière, EspritMadeleine, le prénom de la fille de l'assistant à la réalisation, Philippe Chemin. Isabelle n'était pas le prénom de la fille de Molière.

Ceci est un document de seconde main, qui accrédite les clichés de l'imagerie moliéresque. Comme le tableau du bureau de Jacques Lassalle, la vidéo enregistre et fixe un texte de référence qui est, en grande part, une hagiographie, la légende recomposée par Grimarest, trente ans après la mort de Molière, et accrédite, par exemple, le sort réservé à la traduction du De rerum natura de Lucrèce. L'image de l'autodafé double la référence historique d'une fiction romanesque, celle du Roman de Monsieur de Molière de Mikhaïl Boulgakov, qui sert à cautionner la disparition des manuscrits par un autre autodafé. Boulgakov (p.238 et 252) décrit Molière au soir de sa mort brûlant rageusement un manuscrit. Wilson brûle tous les manuscrits de Molière.

La vidéo collectionne les artefacts de l'imagerie moliéresque. Son fauteuil, ses portraits, mais aussi la collection d'autographes de la Comédie-Française, dont un diptyque qui présente à droite un acte notarié portant un des rares paraphes de Molière, à gauche le brevet de pension accordé aux Comédiens français le 24 août 1682 et signé par Louis XIV et Colbert:

Un petit cadre doré, divisé en deux parties, dont l'une réversible à double face, présente sur le côté fixe de gauche l'original du brevet de 12000 livres allouées aux Comédiens français par Louis XIV. On voit dans le bas, en gros caractères autographes, la signature du Roy-Soleil, et celle de son fidèle ministre Colbert.

[...] Le volet mobile de droite contient, recto et verso, un acte notarié se rapportant à des affaires privées de Molière.

In fine, parmi les noms des contractants, se détache: J.B. Molière, signature authentique du poète offrant aux «chercheurs et curieux» un précieux document d'une valeur inestimable.

(Genest et Duberry, 1922: 255)

La vidéo peut alors convoquer Colbert aux côtés de Molière. L'artefact manuscrit s'incarne dans la présence de Colbert et du couple synecdoque de son effort de peuplement du Canada, la paysanne française et l'Indien d'Amérique. Le diptyque devient triptyque qui met en scène l'américanité. La vidéo est le résultat d'autres circonstances que le cours de 
l'histoire. Le réalisateur fait jouer les artefacts de la collection de la Comédie-Française, qui demeurent indéchiffrables à l'observateur d'images fugitives, comètes du hasard d'un montage. Ceci n'est pas le fruit d'une visite du Musée de la Comédie-Française. La collection de la Comédie-Française n'est pas un sujet de vidéo.

Ceci n'est pas l'histoire de Molière. Personne ne peut écrire l'histoire de Molière. Molière est un objet mythique.

Le film observe le réalisateur qui contrefait l'acteur. Molière mourut à l'âge qu'avait le réalisateur à l'époque du tournage. Wilson s'identifie à Molière qu'il identifie à ses personnages. Molière est Dom Juan. Un «enragé, un chien, un diable, un Turc, un hérétique qui ne croit ni ciel, ni enfer.... un épouseur à toutes mains». Comme lui, il est emporté par les flammes de l'Enfer. Molière est Argan soigné par un moderne Fleurant qui met plus que le nez dans le pot de chambre de son malade.

Müller contrefait le texte de Molière. Il feint la citation, mais la copie est un original, un montage qui déjoue le texte des Femmes savantes, en échangeant les rôles d'Armande et d'Henriette pour forger de toutes pièces le discours de Madeleine Béjart-Armande à Henriette-Armande Béjart.

Le montage joue ainsi sur l'historicité sémantique. Dans la séquence:

Quoi! le beau nom de fille est un titre, ma sœur,

Et sans un mal de cour saurait-on l'écouter?

Qu'a donc le mariage en soi qui vous oblige,

Et de vous marier vous osez faire (la) fête?

les deux premiers vers sont extraits des deux premières répliques de la scène 1 de l'acte I des Femmes savantes. Mais la troisième appartient à la seconde réplique d'Henriette, qui, on se le rappelle, défend le mariage, et s'étonne de voir sa sœur obligée, c'est-à-dire contrainte, par le mariage, alors que le texte de la vidéo l'entend au sens de "qu'est-ce qui vous oblige au mariage»? Le montage joue sur l'interpolation pour faire dire au texte son contraire, son négatif, au sens photographique du terme. Dans la séquence:
Loin d'être aux lois d'un homme en esclave asservie

Et les soins où je vois tant de femmes sensibles

Qui se trouve taillée à faire un philosophe

Si le vôtre est né propre aux élévations

Le Ciel, dont nous voyons que l'ordre est tout puissant

Ne troublons point $d u$ ciel les justes règlements

la plaidoirie féministe - si vous êtes taillée à faire un philosophe, soyez philosophe, sans contrarier la volonté du destin - est tout entière oblitérée par l'interpolation des deux premiers vers qui sont d'Armande et qui retournent le sens des suivants qui sont d'Henriette. L'opposition des deux sœurs Armande était officiellement la sœur de Madeleine devient le discours unique d'un refus du mariage. Refus qui joue autant sur la rivalité de la sœur aînée ou la maîtresse aînée, dépitée de voir la plus jeune épouser celui qui fut son amant pendant de longues années et qui mourut un an, jour pour jour, après elle, que sur l'angoisse de la mère de voir sa fille «tousser et cracher comme elle».

La mort saisit le vif. Madeleine et Armande sont intriquées par le discours, comme elles le furent par les calomnies de l'époque dans une filiation incestueuse que le hors-texte wilsonien annonce en exergue de la séquence.

Le procédé est indéchiffrable, le message brouillé par le mixage de bruits divers. Les dernières paroles ne sont que les vers alternés des répliques d'Henriette et d'Armande, lus à l'envers, mais dont la séquence se lit, à l'endroit, comme suit:

Il n'ait continué ses adorations

Cet empire que tient la raison sur les sens

Et ce n'est pas un fait dans le monde ignoré

Votre esprit à l'hymen renonce pour toujours

Et vous ne tombez pas aux bassesses humaines

Non; mais c'est un dessein qui serait malhonnête

Ainsi reconstituée, la séquence réitère les récriminations d'une femme jalouse, ou même d'une mère torturée par la malhonnêteté d'un hymen qui lie sa fille à son amant.

Ceci est la lecture d'un cryptage. Le cryptage est un décryptage du texte moliéresque qu'il faut, comme les 
paroles des chansons de certains albums des Beatles, lire à l'envers pour révéler les rumeurs qui entourent la vie de Molière.

Il n'eut jamais une autre loy

Que celle qui détruit la foy.

Il se servit de la coquille

Et de la mère et de la fille,

Et ne trouva, dedans sa fin,

Ni Dieu, ni loy, ni medecin. (Donneau de Visé, 1879: 54)

Ceci n'est pas une enquête sur La Mort de Molière. La Mort de Molière n'est pas un sujet d'enquête. La Mort de Molière est un objet pour la sémiotique. D'autres rumeurs de l'époque firent de la mort de Molière une revanche de la médecine. Les contemporains y virent un trophée pour la médecine. Molière était mort en jouant le malade. Il en fut bien puni. Telle est la leçon de maintes épitaphes:

Doctes médecins outragés

Par le satyrique Molière,

La mort, qui l'a mis dans la biere,

Ne vous a-t-elle pas vengés? (Ibid., p.63)

"Quoy! C'est donc le pauvre Molière

Qu'on porte dans le cimetière?"

S'écrièrent quelques voisins.

"Non, dit certain apoticaire:

C'est le Malade imaginaire

Qui veut railler les medecins.» (Ibid., p.52)

La mort, elle-même se fait médecin:

Je n'ai pas, disois-tu, le pouvoir de guérir?

J'ai comme médecin, l'art de faire mourir.

En effet, de ta main, ton avarice extrême,

Jouant le medecin, a creusé ton tombeau.

S'il est du patient l'infaillible bourreau,

Devois-tu devenir medecin toi-même. (Ibid., p.66)

Ceci est un discours sur le danger qu'il y a à se moquer des médecins. La copie risque de devenir l'original. Wilson joue Molière mourant. La copie rejoint l'original et cite une lettre d'un ami du réalisateur l'initiant aux rituels qui tiennent à l'écart la maladie et les médecins. La copie joue à l'original. Ceci n'est pas une pipe.

Ceci est une vidéo. Une vidéo est un objet pour la médecine. Wilson conjure la maladie, mais ne peut conjurer la menace de la médecine. La dernière image de la vidéo, après le générique, est celle du médecin qui fixe la caméra d'un air sardonique ou goguenard. Si la mort de Molière fut, par le biais de poèmes, un sujet de polémique pour ses contemporains, vengeant, entre autres victimes, les médecins des railleries que le dramaturge leur avait fait subir, ne pourrait-elle être, pour Wilson, l'icône propitiatoire de ses propres angoisses?

La mort de Molière fut une pièce à conviction du procès que l'époque faisait aux comédiens qui jouaient à la frontière de la copie et de l'original. Deux termes qui organisent aussi bien la première harangue de Molière devant le Roi, «les comédiens de campagne n'étant que les copies des "originaux" ", comprendre les acteurs de l'Hôtel de Bourgogne, que bon nombre d'épitaphes à lui consacrées.

Cy git qui parut sur la scène

Le singe de la vie humaine

Qui n'aura jamais son égal.

Mais voulant de la mort ainsi que de la vie

Estre l'imitateur dans une comédie,

Pour trop bien réussir, il réussit fort mal;

Car la Mort, en estant ravie,

Trouva si belle sa copie

Qu'elle en fit un original. (Donneau de Visé, 1879: 50)

Cy git Molière! C'est dommage!

Il faisoit bien son personnage;

Il excellait surtout à faire le cocu:

En lui seul, à la Comédie,

Tout à la fois nous avons vu

L'original et la copie. (Ibid., p. 51)

Ci git le singe de la vie,

$\mathrm{Ci}$ git le singe de la mort.

Molière, dans sa Comédie,

Ayant au naturel représenté la mort,

Et par ce dernier acte ayant fini son sort, 
Il passera, malgré l'envie,

pour original sans copie. (Ibid., p.65)

[...]

Mais le plus fort paru au divin tribunal (Monval, 1969: 55)

Où ce paurre insensé, cet ingrat, cet impie,

En pensant de sa mort n'être que la copie,

En devint par malheur le triste original.

Quand Molière, employant de l'art les plus beaux traits,

Nous peignit des humains les differens portraits,

Nous dûmes nos plaisirs à son rare génie;

Mais il ne doit qu'à lui cet honneur sans égal

D'avoir été l'original

Dont La France ne verra jamais la copie.

(Donneau de Visé, 1879: 68)

Dans le contexte de la modernité, seul l'original est apprécié. Wilson fait œuvre originale. Pourtant, il reprend bon nombre des clichés qui structurent la légende moliéresque. Copie de la copie, il ne peut s'adresser qu'à un public pour qui l'original n'existe pas. Le repère ou le référent sont sans importance au regard de l'image ou du cliché: on peut faire nettoyer par le père le fauteuil du fils devenu, le temps d'un "Father, I am diying», l'image du crucifié. Le substrat historique est emprunté par Wilson aux commères de l'histoire (Grimarest, Boulgakov), à quelques chromos, aux métaphores les plus connues des Fables de La Fontaine (le renard). La culture s'énonce dans le galimatias qu'est devenu le latin, le grec, dans la ribambelle des religieuses découpée sur le modèle des deux religieuses de l'époque.
Ceci est une vidéo. Ce n'est pas une vidéo sur Molière. Molière n'est pas un sujet de vidéo. Molière est un objet pour le théâtre. Que les athées et les impies de l'imagerie postmoderne se le tiennent pour dit et méditent sémiotiquement cette dernière épitaphe:

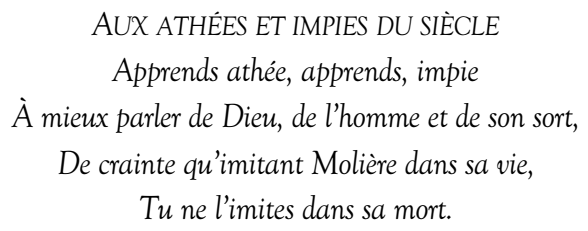

(Donneau de Visé, 1879: 68)

\section{RÉFÉRENCES BIBLIOGRAPHIQUES}

BOUlGaKOV, M. [1973]: Le Roman de Monsieur de Molière, Paris, Éd. Champ libre.

DONNEAU DE VISÉ, J. [1879]: Oraison funèbre de Molière, Paris, Librairie des bibliophiles, Genève, Slatkine Reprints, coll. «Nouvelle collection moliéresque".

DUCHÊNE, R. [1998]: Molière, Paris, Fayard.

Genest, G. et É. DuberRy [1922]: La Maison de Molière connue et inconnue, Paris, Librairie Fischbacher.

JÜRGENS, M. et E. MAXFIELD-MILLER [1963]: Cent ans de recherches sur Molière, Paris, S.E.V.P.E.N.

LINDERS, J. [1996] : «Molière \pm Müller », Performance Research, vol. 1, $\mathrm{n}^{\circ} 2,93-102$.

LORCEY, J. [1980]: La Comédie française, Paris, Fernand Nathan. MONVAL, G. [1969]: Recueil sur la mort de Molière, Genève, Slatkine Reprints, coll. «Nouvelle collection moliéresque». 
Chère E.L.

Ai trouvé par hasard en bouquinant au bord de la Seine cette photographie présumée mais sans doute fausse de Molière. L'ai tout de même achetée... sait-on jamais. Wish you were here.

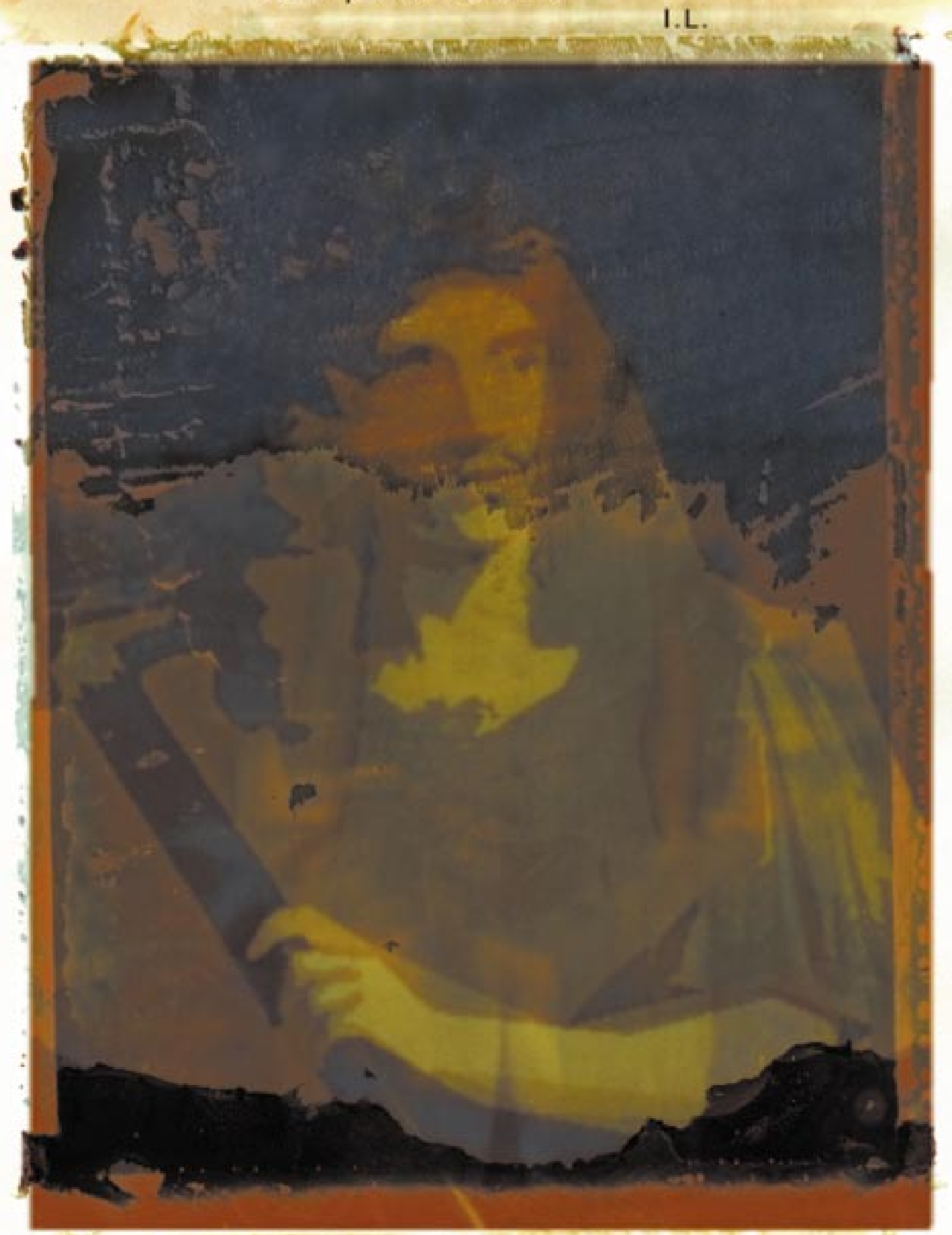

Carol Dallaire, Souvenir de Paris, 1999.

À partir de Molière dans le rôle de César par Mignard (collections Comédie-Française). 